\title{
Sink-Convergence Cascading Model for Wireless Sensor Networks with Different Load-Redistribution Schemes
}

\author{
Xiuwen Fu $\mathbb{D}$, Haiqing Yao $\mathbb{D}$, and Yongsheng Yang $\mathbb{D}$ \\ Institute of Logistics Science and Engineering, Shanghai Maritime University, Shanghai 201306, China \\ Correspondence should be addressed to Xiuwen Fu; fuxiuwen1987@163.com
}

Received 27 December 2018; Revised 22 April 2019; Accepted 28 May 2019; Published 19 June 2019

Guest Editor: Riccardo Patriarca

Copyright (C) 2019 Xiuwen Fu et al. This is an open access article distributed under the Creative Commons Attribution License, which permits unrestricted use, distribution, and reproduction in any medium, provided the original work is properly cited.

Existing cascading models are unable to depict the sink-convergence characteristic of WSNs (wireless sensor networks). In this work, we build a more realistic cascading model for WSNs, in which two load-redistribution schemes (i.e., idle redistribution and even redistribution) are introduced. In addition, failed nodes are allowed to recover after a certain time delay rather than being deleted from the network permanently. Simulation results show that the network invulnerability is positively correlated to the tolerance coefficient and negatively correlated to the exponential coefficient. Under the idle-redistribution scheme, the network can have stronger invulnerability against cascading failures. The extension of the recovery time will exacerbate the fluctuation of the cascading process.

\section{Introduction}

Wireless sensor network (WSN) is one of the most important components of the Internet of Things (IOT) system, because it has the characteristics of simple deployment, low cost, selforganization, and so on $[1,2]$. In actual WSNs, sensor nodes are characterized by limited capacity. If the traffic load of a sensor node is greater than its capacity, its performance will be severely affected and all or part of its load will be rerouted to other sensor nodes, further leading to a redistribution of traffic load across the network. During this process, there may be new sensor nodes being failed due to overload. We call this dynamic process the cascading failures. In WSNs, due to the existence of cascading failures, even though most failures emerge very locally, the entire network can be largely affected or even collapsed globally [3-5].

Existing cascading models for WSNs usually used the degree or betweenness value of a sensor node to represent their traffic load. These assumptions are reasonable enough in the peer-to-peer networks, but they cannot apply to WSNs as they ignored the impacts of the sink node on network traffic distribution. Sink convergence is the most evident characteristic that can distinguish WSNs from other networks. Therefore, this paper proposes a more realistic cascading model for WSNs. The main contributions of this paper are as follows:

(1) A cascading model that can depict the sink-convergence characteristic of WSNs is proposed.

(2) Two load-redistribution schemes (i.e., even-redistribution scheme and idle-redistribution scheme) are introduced.

(3) We evaluate the impacts of key parameters in this model and compare two load-redistribution schemes.

The reminder of the paper is organized as follows. Section 2 describes recent related work. In Section 3, the cascading model is proposed. In Section 4, simulation results are given. Finally, conclusion and the future work are presented.

\section{Related Work}

In the real world, cascading failures are very common in actual network systems, such as power grid network, supply chain network, and communication network. Many researchers attempted to model the cascading process of actual networks [6]. Wang et al. [7] developed an under-load cascading model of supply chain networks, where each node is characterized by a capacity with upper and lower bounds. Rohden et al. [8] studied the cascading invulnerability of 
TABLE 1: Summary of existing cascading models.

\begin{tabular}{lccccc}
\hline Models & Network types & Load Metrics & Models & Network types & Load Metrics \\
\hline$[7]$ & supply chain networks & degree & {$[14]$} & WSNs & betweenness \\
{$[8]$} & electricity grids & simulated current & {$[15]$} & WSNs & degree \\
{$[9]$} & communication networks & betweenness & {$[16]$} & WSNs & betweenness \\
{$[10]$} & interdependent networks & betweenness & {$[17]$} & WSNs & exponential degree \\
{$[11]$} & transmission networks & betweenness & {$[18]$} & WSNs & betweenness \\
{$[12]$} & cyber-physical systems & betweenness & {$[19]$} & WSNs & cluster degree \\
{$[13]$} & transportation networks & degree & {$[20]$} & WSNs & number of messages \\
\hline
\end{tabular}

electricity grids based on the alternating current model. Ren et al. [9] proposed a stochastic model to study the cascading dynamics in communication networks and identified the vital nodes from the perspective of network invulnerability. Chen et al. [10] investigated the cascading process in interdependent power grids and communication networks. Wu et al. [11] analyzed the impacts of link capacity on the cascading process in general transmission networks and found that a bifurcation point may exist in some cases which divides regions of opposite robustness behavior. Tu et al. [12] investigated the cascading invulnerability of cyberphysical systems and observed that two coupling networks have different sensitivity to the failure propagated from the other network. Candelieri et al. [13] investigated the cascading invulnerability of public transportation networks against directed attacks.

WSNs have also received a lot of attention in terms of cascading failures. Liu et al. [14] proposed a betweennessoriented cascading model. In this model, the traffic of a sensor node is defined as its betweenness value. Yin et al. [15] studied the cascading process of scale-free WSNs and assumed that the traffic load of sensor nodes is correlated to their degrees. Li et al. [16] used the probability generation function to analyze the critical load of scale-free WSNs. In this work, the load is set to be closely correlated to the betweenness value. Ye at al. [17] proposed a fault-tolerant scheme to resist cascading failures in WSNs. They assumed that the load of sensor nodes is correlated to their degrees in an exponential way. $\mathrm{Hu}$ at al. [18] analyzed the cascading process of WSNs under random attacks based on the betweenness-load model. In [19], we presented a cascading model for hierarchical WSNs. In this model, the nodes' load is determined by its intercluster degree and its inner-cluster degree. In [20], we proposed a routingbased cascading model of WSNs in which the load of sensor nodes is defined as the real-time number of messages they carry.

Table 1 summarizes the mentioned cascading models. Although many cascading models have been proposed, they do not apply to realistic WSNs because they cannot reflect the sink-convergence characteristic of WSNs. A sample of sink convergence in WSNs is shown in Figure 1. In realistic WSNs, all the data packets collected by general sensor nodes will eventually be collected at the sink node and then be uploaded to the cloud; thus WSNs follow a typical many-toone transmission paradigm, which makes them exhibit completely different traffic characteristics from other networks.

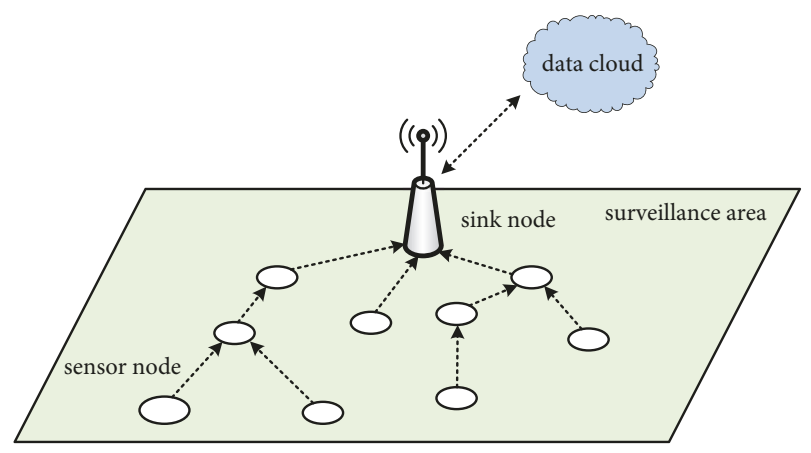

FIGURE 1: A sample of sink convergence in WSNs.

Therefore, it is necessary to develop a more realistic cascading model of WSNs.

\section{Cascading Model}

3.1. Traffic Metric. In [21], we have proposed a traffic metric "sink-oriented betweenness" to characterize the load distribution of WSNs. Its effectiveness and soundness have been verified through extensive simulations in [21]. Therefore, in this work we still use this traffic metric, as described below:

$$
C_{i}(t)=\frac{\sum_{j \in V} g_{i, j}(t) / g_{j}(t)}{N},
$$

where $g_{i, j}(t)$ is the number of the shortest paths from node $j$ to the sink node passing through node $i$ at time $t . g_{j}(t)$ is the number of the shortest paths from node $j$ to the sink node at time $t . V$ and $N$ are the set of sensor nodes and the total number of sensor nodes in the network, respectively.

3.2. Load and Capacity. As discussed in the last section, in actual WSNs, sensor nodes' initial load is correlated to the number of shortest paths from all the other sensor nodes to the sink node passing through it in the network, so it is reasonable to define the nodes' initial load as a function of the sink-oriented betweenness. For this consideration, we define the initial load of node $i$ as

$$
L_{i}(0)=C_{i}(0)^{\alpha},
$$

where $\alpha \geq 0$ is the load-exponential coefficient that determines the distribution of the initial load. We can easily 
observe that the initial load of each sensor node bears a linear relationship with its sink-oriented betweenness value when $\alpha=1$. The configuration of $\alpha$ is closely related to the data type of WSNs. If the data type is the multimedia data, it means that the initial load will have a rapid growth in an exponential way with the increase of $C_{i}(0)$; thus $\alpha$ should be set to a relatively large value. If the data type is the general text data, $\alpha$ can be a small value. It is obvious that the introduction of $\alpha$ can provide a high flexibility for our model to apply to different types of WSNs.

In most literature $[14,16]$, the nodes' capacity is set to be positively correlated to their initial load, as shown in

$$
W_{i}=(1+\beta) L_{i}(0),
$$

where $\beta$ is the overload-tolerance coefficient. However, in WSNs, this setting is far from the realistic situations. Unlike power grids in which the nodes' capacity can be customized according to the practical demands, the nodes' capacity in WSNs is always the same. This is partly because in most cases the hardware configurations of sensor nodes within the same WSN are always the same, and partly because it is impossible to customize the nodes' capacity when hundreds and even thousands of them are deployed. Therefore, in this work, the sensor nodes' capacity is defined as

$$
W_{N}=(1+\beta) L_{N}(0)=(1+\beta) \frac{\sum_{i=1}^{N} L_{i}(0)}{N} .
$$

According to (4), each sensor node has the same capacity, which is positively correlated to the average load of the initial network.

3.3. Load-Redistribution Schemes. In case that node $i$ fails, its load will be distributed to other nodes in the network. There are two load-redistribution schemes: (1) evenredistribution scheme; (2) idle-redistribution scheme. The even-redistribution scheme is widely used in many cascading models. Under this scheme, the load originally taken by the failed node will be redistributed to its neighboring nodes. If node $i$ fails at time $t$, its neighbor $j$ can receive extra load $\Delta_{j i}$ at time $t+1$ as follows:

$$
\Delta_{j i}(t)=\frac{1}{N_{i}(t)} L_{i}(t)
$$

where $N_{i}(t)$ is the number of neighbors that node $i$ has at time $t$. In some routings protocols of WSNs, sensor nodes do not have the real-time state information about their neighbors and they have the same capacity. It is reasonable to assign the load of the failed node to its neighbors evenly.

With the development of routing technologies in WSNs, in some routing protocols, sensor nodes can be congestionaware, which means that they can own the real-time state information regarding their neighbors. On this basis, the idleredistribution scheme is proposed. If node $i$ fails at time $t$, its neighbor $j$ can receive extra load $\Delta_{j i}$ at time $t+1$ as follows:

$$
\Delta_{j i}(t)=\frac{W_{N}-L_{j}(t)}{\sum_{k \in \Omega_{i}(t)}\left[W_{N}-L_{k}(t)\right]} L_{i}(t),
$$

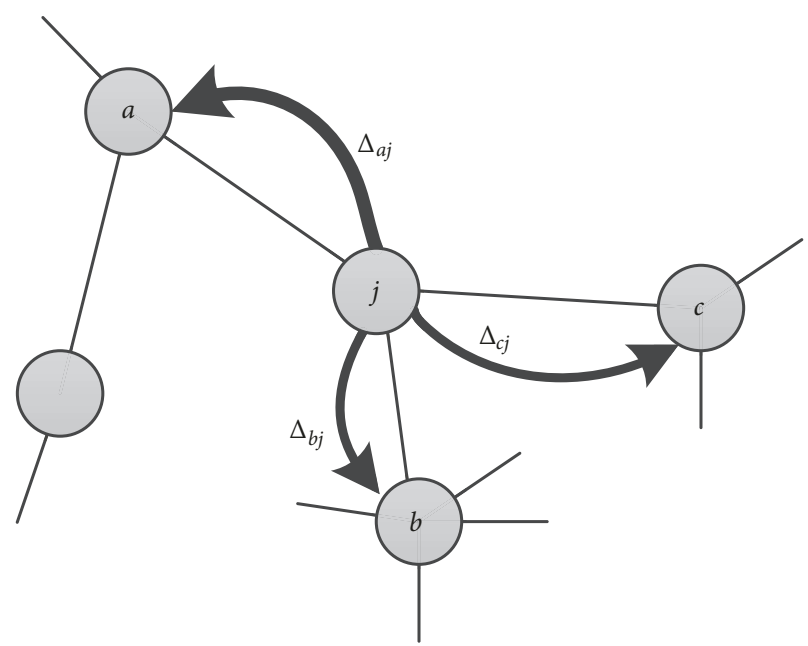

FIGURE 2: An example of load-redistribution process.

where $\Omega_{i}(t)$ is the set consisting of the neighbors of node $i$ at time $t$. $W_{N}-L_{k}(t)$ is the idle capacity of node $k$, which can also be understood as the maximum load it can still receive. According to (6), we can easily find that, under the idleredistribution scheme, the node with more idle capacity can be assigned more load from the failed node.

To illustrate the load-redistribution process more clearly, we present an example on a simplified network topology (shown in Figure 2). Assuming that node $j$ fails at time $t$, the original load it takes will transfer to its neighbors $a, b$, and $c$ according to the load-redistribution scheme. At time $t+1$, the real-time load of nodes $a, b$, and $c$ will be updated according to (7).

$$
\begin{aligned}
& L_{a}(t+1)=L_{a}(t)+\Delta_{a j} \\
& L_{b}(t+1)=L_{b}(t)+\Delta_{b j} \\
& L_{c}(t+1)=L_{c}(t)+\Delta_{c j} .
\end{aligned}
$$

If $L_{i}(t+1)>W_{N}, i \in\{a, b, c\}$, another round of node failures will be triggered and the load of the newly failed node will transfer to its neighbors. This cascading process will not stop until the load of remaining nodes is within their capacity.

3.4. Cascading Mechanism. In most of the existing cascading models, sensor nodes have two states: normal and overloaded. According to their assumptions, if the node's load is beyond its capacity, then it will be removed from the network permanently. This assumption is reasonable in the network like power grids. However, in WSNs, this assumption is far from the fact. Different from the electricity overload in power grids, the overload of data packets in WSNs will not cause physical damage of sensor nodes. Overloaded nodes will reboot rather than fail permanently. When the reboot is completed, it will join the network again and function normally. Thus, in our model, the node at overloaded state will be given a recovery time $\Delta t$. Within $\Delta t$, this node cannot 


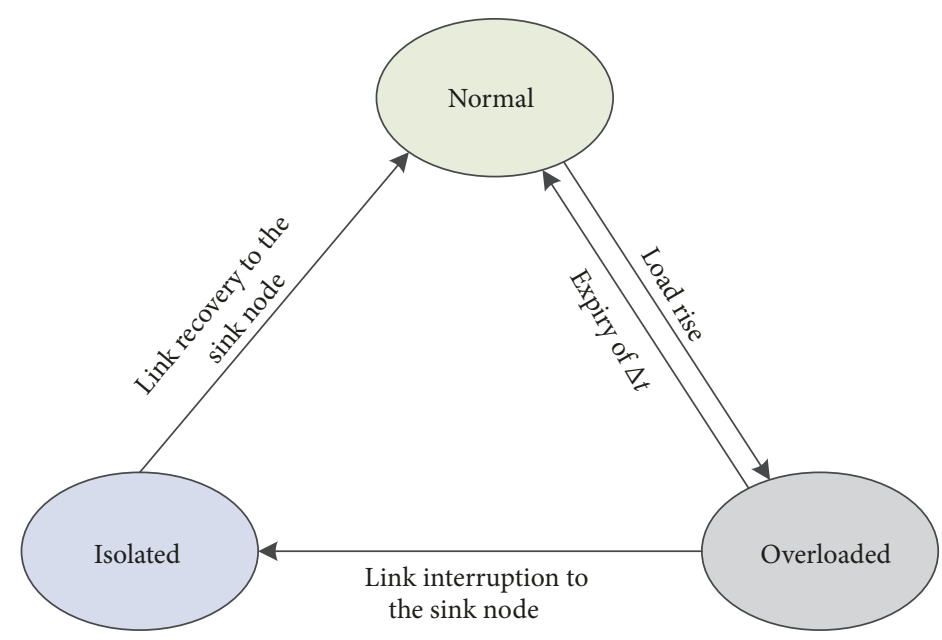

FIGURE 3: Cascading-state transition of sensor nodes.

receive, process, and transmit data packets. When $\Delta t$ is expired, the node will become "normal" again. It is easy to understand that overloaded node can be recovered instantly when $\Delta t$ approaches 0 . Apparently, in this case, the damage caused by overload can be minimized. If $\Delta t$ approaches $\infty$, our cascading scheme is equivalent to the conventional "permanent removal" scheme.

Sink convergence is the most evident feature that distinguishes WSNs from other wireless networks. In WSNs, if the link between a sensor node and the sink node is interrupted, the sensor node will be considered as an isolated node as its messaging service is not available. When cascading failures occur, some sensor nodes will become overloaded and the network connectivity will be severely impaired. During this process, some nodes will be isolated as their paths to the sink nodes are cut off. When some overloaded nodes are recovered via reboot, the network connectivity can be restored and isolated nodes will return to normal. The state transitions in the cascading mechanism is shown in Figure 3.

\section{Analysis of the Invulnerability of WSNs}

4.1. Simulation Settings. In the simulations, we set the network size to 300 and sensor nodes are randomly deployed in the simulation area. The wireless transmission radius of sensor nodes is set to $20 \mathrm{~m}$ and the sink node is placed at the center of the simulation area. Figure 4 shows the network topology. In order to trigger cascading failures, we initially attack the first $10 \%$ of sensor nodes in the descending order of sink-oriented betweenness. Each node in the initial network before attack is at the normal state. We use survival ratio $H_{n}(t)$ to measure the network invulnerability against cascading failures. As discussed in Section 3.4, normal nodes are the nodes that are not overloaded and can still maintain at least one effective path to the sink node. $H_{n}(t)$ can be calculated by

$$
H_{n}(t)=\frac{N(t)}{N(1-q \%)}
$$

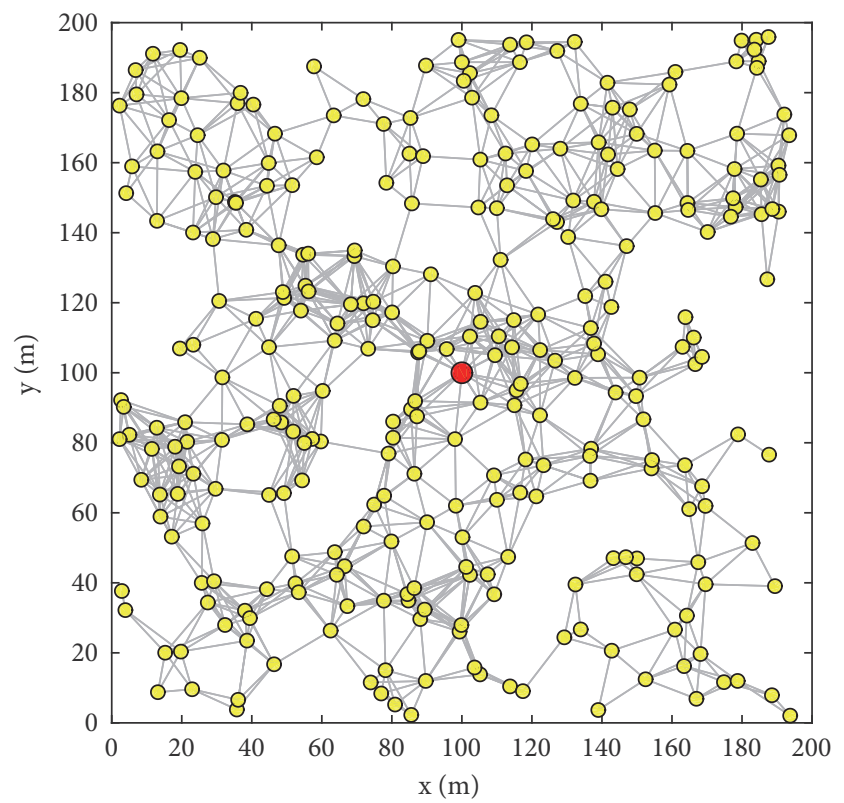

FIGURE 4: Network topology (300 sensor nodes are deployed and the sink node is marked in red).

where $N(t)$ is the number of normal nodes at time $t$. Here we use $H_{n}(\infty)$ to represent the survival ratio when the network reaches the steady state.

\subsection{Simulation Results}

4.2.1. Verification of Sink-Convergence Characteristic. The purpose of this experiment is to verify the sink-convergence characteristic of the proposed cascading model. In actual WSNs, since the sensor nodes around the sink node need to undertake more message-forwarding tasks, their load will be significantly higher than that of the nodes far from the sink 


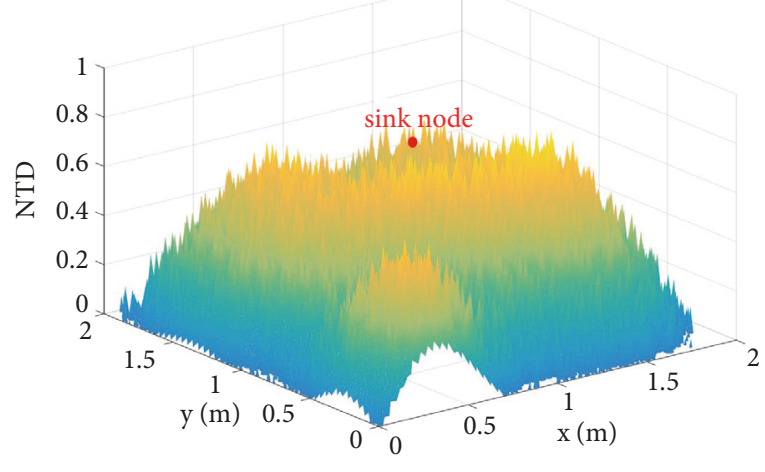

(a) Degree

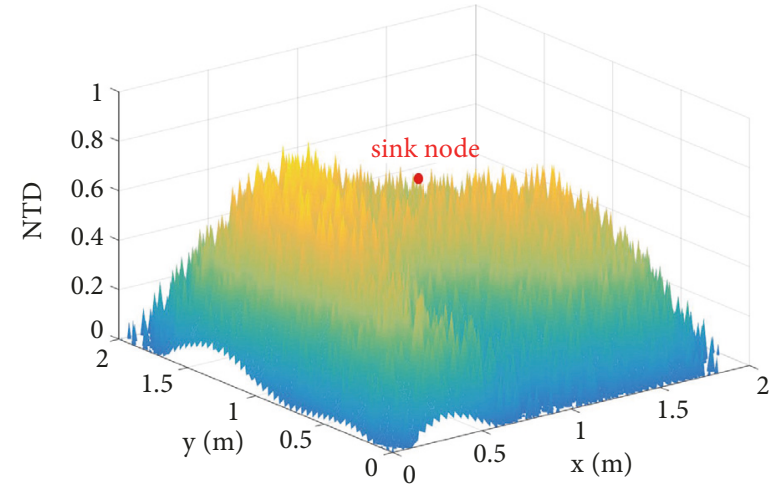

(b) Betweenness

FIGURE 5: Network traffic distribution (NTD) generated by the degree-based and betweenness-based cascading models, respectively.

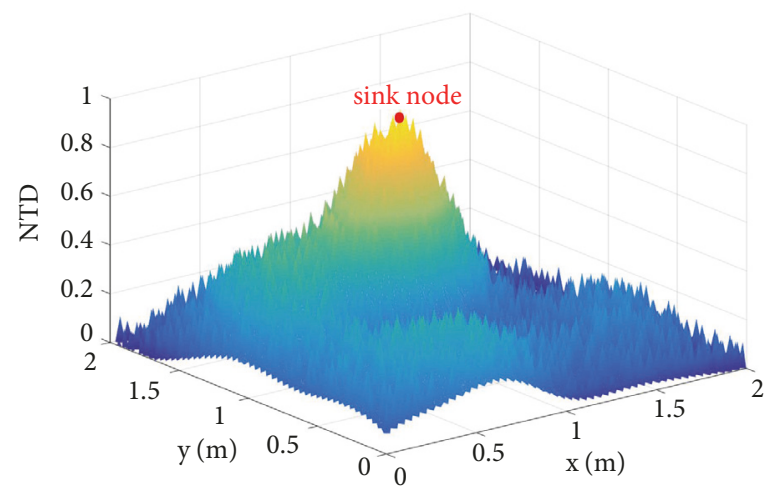

Figure 6: Network traffic distribution (NTD) generated by the proposed cascading model $(\alpha=1)$.

node, a phenomenon described by many researchers as the "sink hole" [22-26]. Apparently, the sink hole phenomenon is an important indicator for judging whether the network is characterized by sink convergence.

Figure 5 shows the network traffic distribution created by the degree-based cascading model and the betweennessbased cascading model, respectively. It can easily observed that there is no significant difference between the load of the nodes around the sink node and the load of the nodes in other areas, so the energy hole phenomenon is not so obvious. Figure 6 shows the network traffic distribution created by the proposed cascading model. We can easily observe a highload peak around the sink node, so the sink-convergence characteristic is verified.

4.2.2. Impacts of Modeling Parameters. As is shown in Figure 7, we can easily find that, with the increase of exponential coefficient $\alpha, H_{n}(t)$ tends to decrease and the cascading process will reach the steady state faster. For example, under the even-redistribution scheme, when $\alpha=0.5, H_{n}(t)$ will reach the steady state at $81 \%$ at time $t=3$. When $\alpha$ rises to $2, H_{n}(t)$ will stabilize at $17 \%$ at time $t=5$. It is easy to understand that the load taken by sensor nodes will increase much faster in an exponential way with the growth of $\alpha$, which will lead to a more evident gap between low-load nodes and high-load nodes. When the high-load nodes are attacked, the low-load nodes can hardly have enough capability to tackle the extra load transferred from failed high-load nodes. In our model, the configuration of $\alpha$ is closely related to the data type of WSNs. The above simulation results tell us that for the high-volume data type, the risks and the damage brought by cascading failures will be much higher and the network designer should pay more attention to prevention of cascading failures.

Through the comparison between Figures 7(a) and 7(b), we can also find that the idle-redistribution scheme demonstrates a stronger invulnerability than the even-redistribution scheme when facing cascading failures. In the case that $\alpha=1$, under the even-redistribution scheme and idle-redistribution scheme, $H_{n}(t)$ will stabilize at $47 \%$ and $83 \%$, respectively. This is because under the idle-redistribution scheme, the idle capacity can be fully used, and thus more load can be tackled.

From Figure 8, we can find that the network invulnerability can be significantly improved with the increase of overload-tolerance coefficient $\beta$. In our model, a higher $\beta$ means that sensor nodes can have more capacity to tackle load. Thus, there is surely a threshold $\beta^{*}$ that can provide enough capacity for sensor nodes and can protect them from being overloaded. From Figure $8(\mathrm{~b})$, we can find that the cascading process under $\beta=1.5$ and $\beta=2$ is totally the same. This phenomenon tells us that the threshold $\beta^{*}$ should be within $[1,1.5]$.

Figure 9 depicts the composition of failed nodes when the network reaches the steady state. We can clearly find that although neighboring nodes being overloaded constitute the major reason that makes nodes isolated, the majority of failed nodes are isolated nodes. Under the even-redistribution scheme, in the case that $\beta=0.5$, isolated nodes are $63 \%$ and overloaded nodes are $21 \%$. Moreover, with the increase of $\beta$, the ratio of overloaded nodes tends to be smaller. When $\beta$ reaches a certain value, there will be no overloaded 


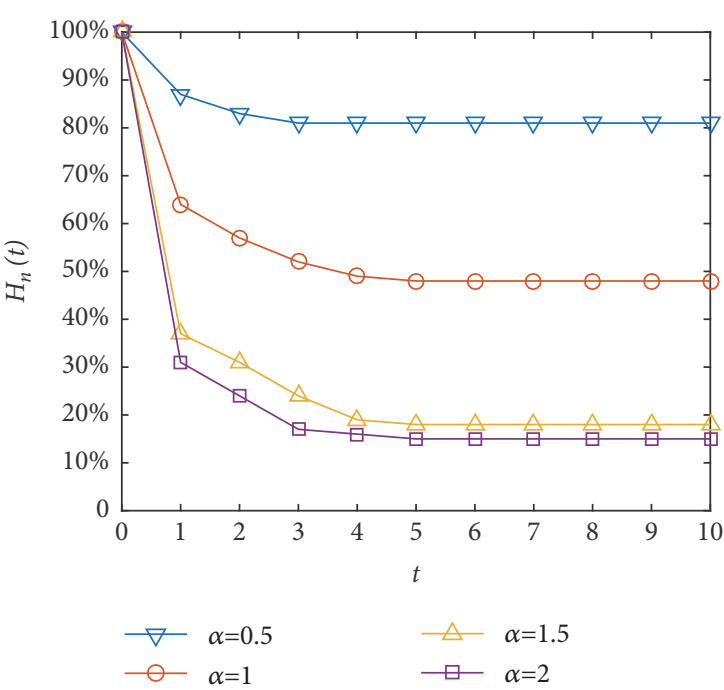

(a) Even-redistribution scheme

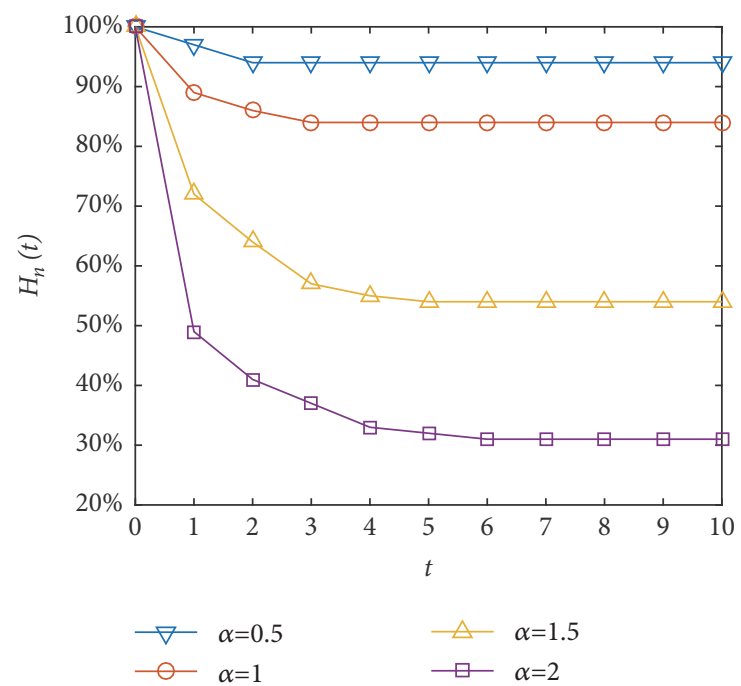

(b) Idle-redistribution scheme

Figure 7: Survival ratio with varying $\alpha(\beta=1, \Delta t=\infty)$.

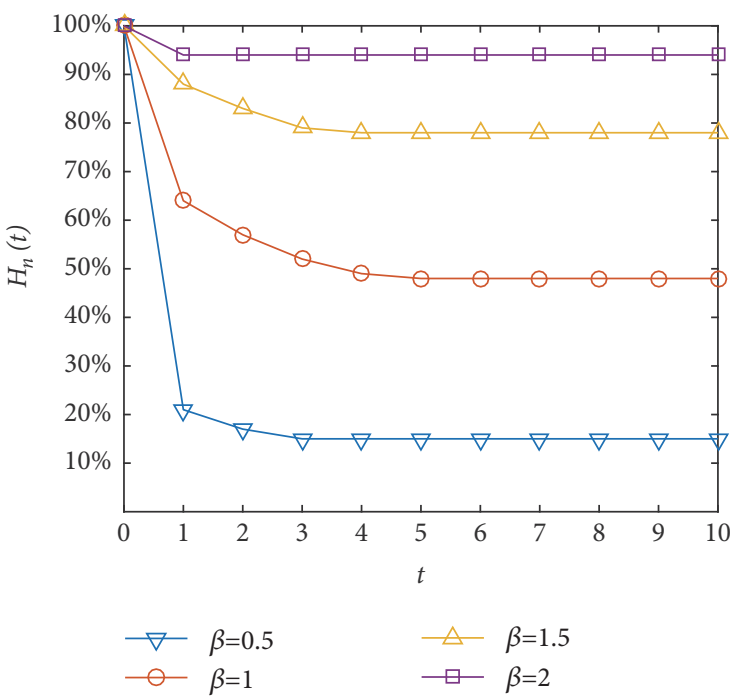

(a) Even-redistribution scheme

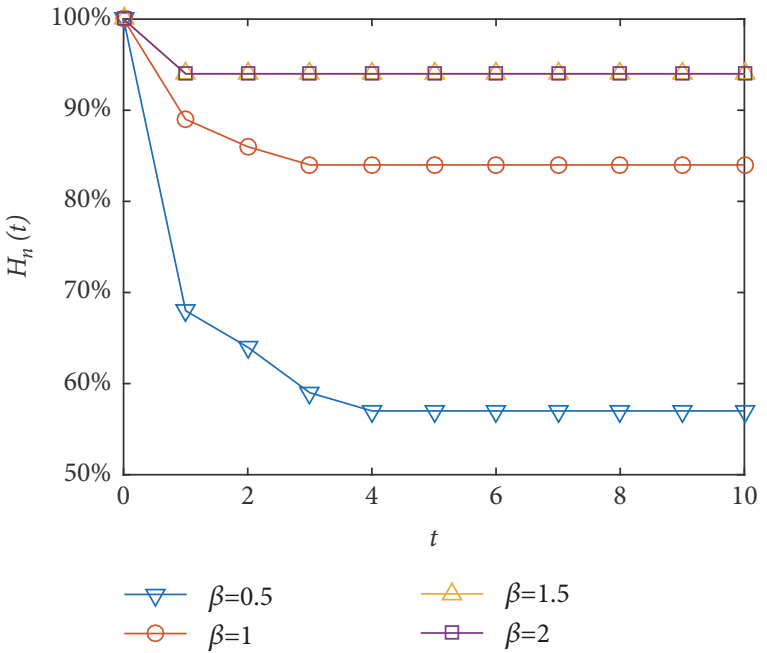

(b) Idle-redistribution scheme

FIGURE 8: Survival ratio with varying $\beta(\alpha=1, \Delta t=\infty)$.

nodes in the network, which means the cascading process will not be triggered and the only damage is the isolated nodes caused by initial attacks. The existence of $\beta^{*}$ is further verified.

As is shown in Figure 10, it can be easily observed that the threshold $\beta^{*}$ will increase with the growth of $\alpha$, which means that more capacity resources are required to protect the network from cascading failures. Through comparison between Figures 10(a) and 10(b), we can find that at the same settings $\beta^{*}$ will be smaller under the idle-redistribution scheme than under the even-redistribution scheme. The advantages of idle-redistribution scheme are further verified.
Figure 11 depicts the impacts of recovery time $\Delta t$ on survival ratio $H_{n}(t)$. It can be easily observed that $H_{n}(t)$ tends to fluctuate more wildly with the increase of $\Delta t$. In the case of $\Delta t=1$, when some sensor nodes are overloaded, on the one hand, they will redistribute their load and cause some other nodes to overload in the next time step and, on the other hand, they can recover from overload at the next time step due to the expiry of $\Delta t$. Therefore, we can find that at each time step after $t=2$, some nodes in the network fall into failure and some nodes return to normal, which makes $H_{n}(t)$ demonstrate slight fluctuations with the network cascading process. Although in this case the damage caused by cascading failures can be minimized, it is actually hard to 


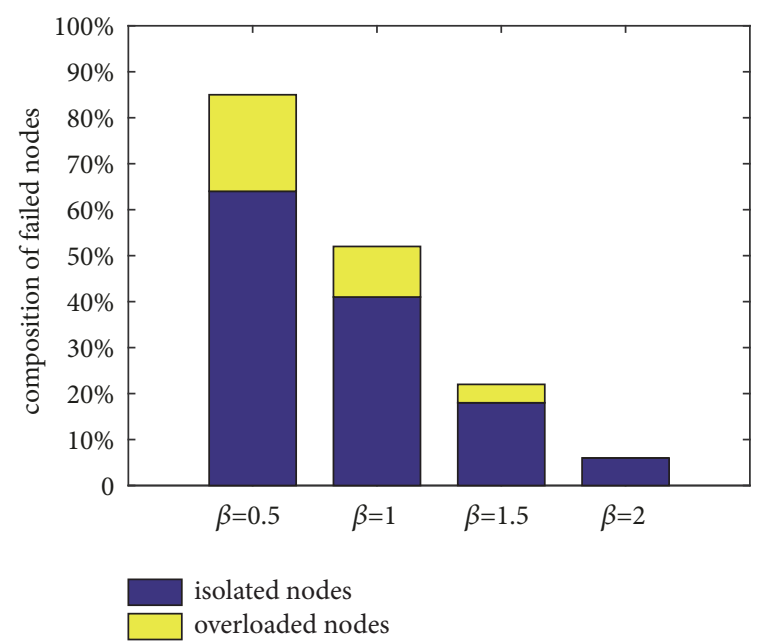

(a) Even-redistribution scheme

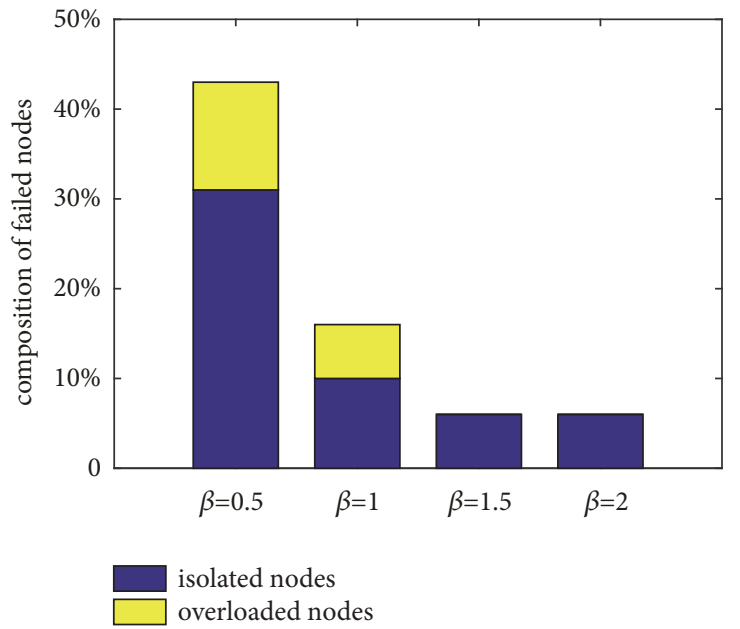

(b) Idle-redistribution scheme

FIGURE 9: Composition of failed nodes with varying $\beta(\alpha=1, \Delta t=\infty)$.

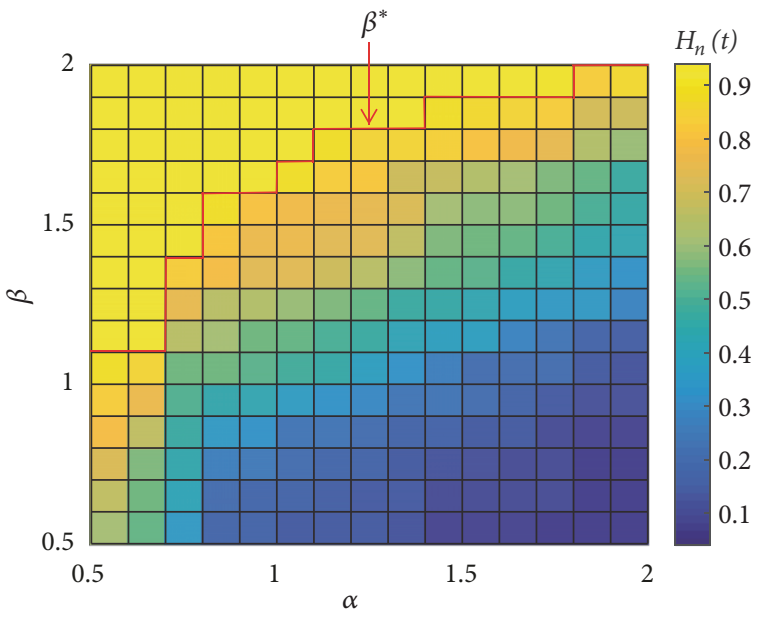

(a) Even-redistribution scheme

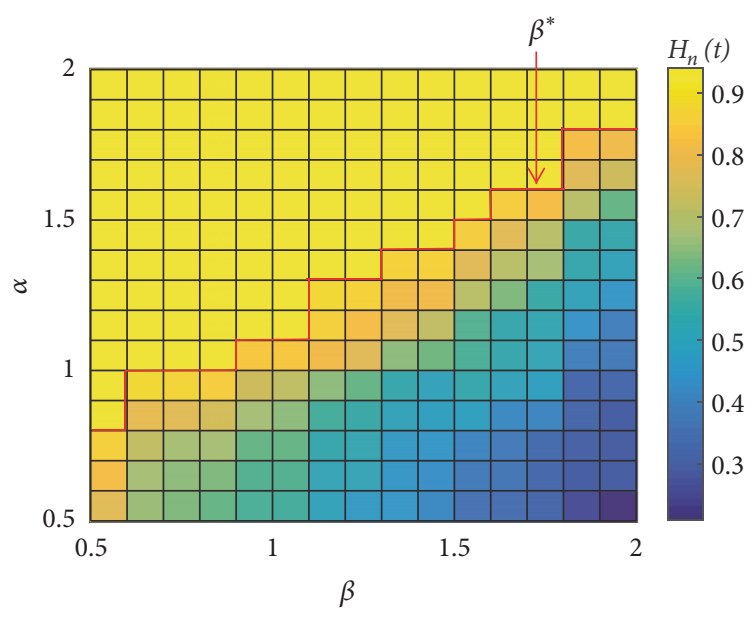

(b) Idle-redistribution scheme

Figure 10: Heatmap of $H_{n}(\infty)$ in the parameter space $[\alpha, \beta]$ (threshold $\beta^{*}$ is labelled by the red curve).

achieve because sensor nodes take time to reboot. When $\Delta t$ increases to 2 or 3 , overloaded nodes require more time for recovery, which will make cascading failures spread to a wider range and then lead to more obvious fluctuations of $H_{n}(t)$. When $\Delta t=\infty$, sensor nodes lose the recovery ability and $H_{n}(t)$ decreases monotonically to a steady-state value.

\section{Conclusions}

In this paper, we developed a more realistic cascading model for WSNs. The most significant advantage of this model is that it can properly reflect the sink-convergence characteristic of WSNs. The simulation results show that (1) the network invulnerability is positively correlated to the overloadtolerance coefficient and negatively correlated to the loadexponential coefficient; (2) under the idle-redistribution scheme, the network can have stronger invulnerability against cascading failures; and (3) the extension of the recovery time will exacerbate the fluctuation of the cascading process. These results provide us with some meaningful guidelines to build a more invulnerable WSN against cascading failures.

(1) The network with high-volume data type is more vulnerable to cascading failures.

(2) Due to the advantages of the idle-redistribution scheme, congestion-aware routing protocols can tackle more load, thus gaining stronger invulnerability against cascading failures.

In this work, we only discuss the cascading invulnerability of WSNs with deploying only one sink node. In recent years, multisink WSNs are becoming more and more widely used due to their advantages in energy efficiency and load balancing. Therefore, in our future work, we hope to upgrade the proposed model to adapt to the multisink WSNs, and on this basis, study its cascading invulnerability. 


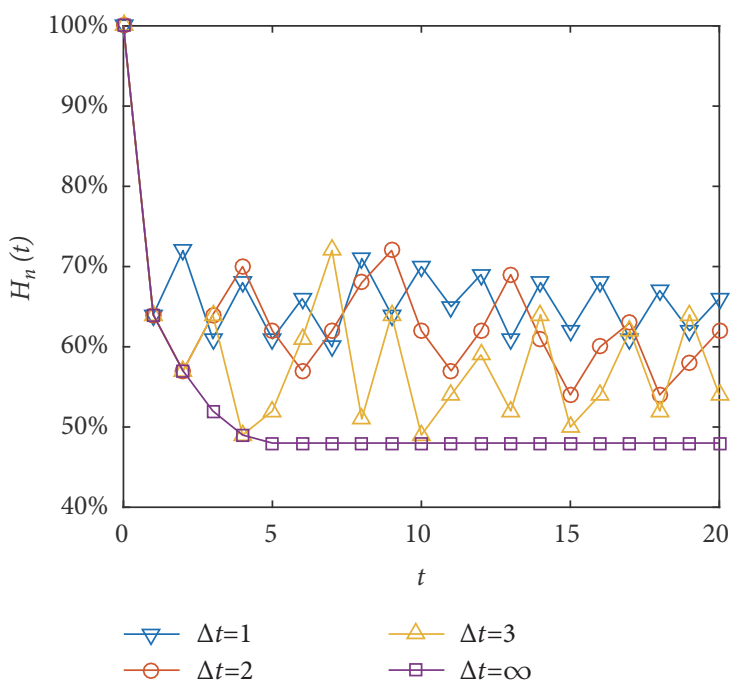

(a) Even-redistribution scheme

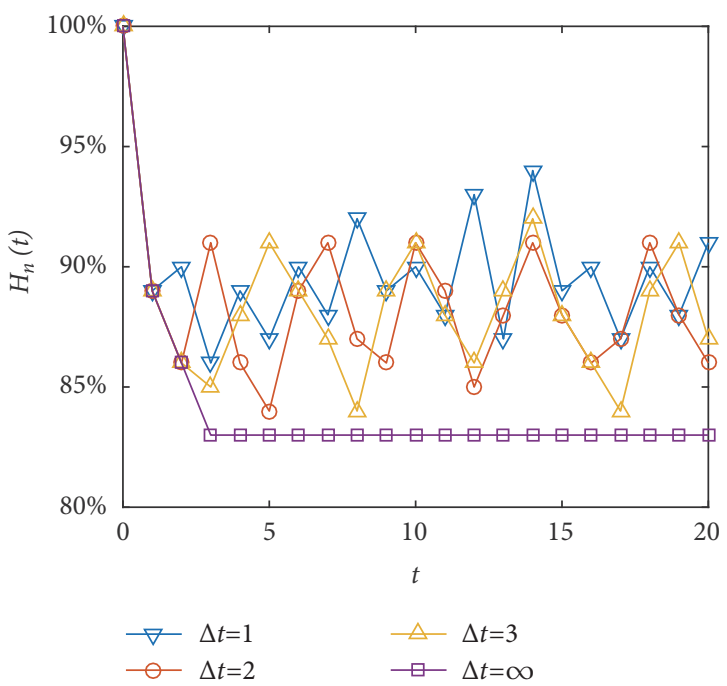

(b) Idle-redistribution scheme

FIGURE 11: Survival ratio with varying $\Delta t(\alpha=\beta=1)$.

\section{Data Availability}

The data used to support the findings of this study are available from the corresponding author upon request.

\section{Conflicts of Interest}

The authors declare that there are no conflicts of interest regarding the publication of this article.

\section{Acknowledgments}

This work is supported in part by the National Natural Science Foundation of China (NSFC) under Grant No. 61571336.

\section{References}

[1] Y. Duan, X. Fu, W. Li, Y. Zhang, and G. Fortino, "Evolution of scale-free wireless sensor networks with feature of small-world networks," Complexity, vol. 2017, Article ID 2516742, 15 pages, 2017.

[2] X. Fu, Y. Yang, and H. Yao, "Analysis on invulnerability of wireless sensor network towards cascading failures based on coupled map lattice," Complexity, vol. 2018, 14 pages, 2018.

[3] X. Fu, H. Yao, O. Postolache, and Y. Yang, "Message forwarding for WSN-Assisted Opportunistic Network in disaster scenarios," Journal of Network and Computer Applications, vol. 137, pp. 11-24, 2019.

[4] X. Fu, G. Fortino, and W. Li, "Environment-cognitive multipath routing protocol in wireless sensor networks," in Proceedings of the 2018 IEEE International Conference on Systems, Man, and Cybernetics (SMC), pp. 2760-2765, IEEE, Miyazaki, Japan, October 2018.

[5] G. Fortino, W. Russo, C. Savaglio, W. Shen, and M. Zhou, "Agent-oriented cooperative smart objects: from iot system design to implementation," IEEE Transactions on Systems Man \& Cybernetics Systems, no. 99, pp. 1-18, 2017.

[6] T. Wu, J. Wu, and W. You, "Optimizing robustness of complex networks with heterogeneous node functions based on the memetic algorithm," Physica A: Statistical Mechanics and its Applications, vol. 511, pp. 143-153, 2018.

[7] Y. Wang and F. Zhang, "Modeling and analysis of under-loadbased cascading failures in supply chain networks," Nonlinear Dynamics, vol. 92, no. 3, pp. 1403-1417, 2018.

[8] M. Rohden, D. Jung, S. Tamrakar, and S. Kettemann, "Cascading failures in ac electricity grids," Physical Review E, vol. 94, no. 3, Article ID 032209, 2016.

[9] W. Ren, J. Wu, X. Zhang, R. Lai, and L. Chen, "A stochastic model of cascading failure dynamics in communication networks," IEEE Transactions on Circuits and Systems II: Express Briefs, vol. 65, no. 5, pp. 632-636, 2018.

[10] Z. Chen, J. Wu, Y. Xia, and X. Zhang, "Robustness of interdependent power grids and communication networks: a complex network perspective," IEEE Transactions on Circuits and Systems II: Express Briefs, vol. 65, no. 1, pp. 115-119, 2018.

[11] J. Wu, X. Zhang, S. Ma, Z. Rong, and C. K. Tse, "Bifurcation in transmission networks under variation of link capacity," International Journal of Bifurcation and Chaos, vol. 28, no. 9, Article ID 1850109, 2018.

[12] H. Tu, Y. Xia, J. Wu, and X. Zhou, "Robustness assessment of cyber-physical systems with weak interdependency," Physica A: Statistical Mechanics and its Applications, vol. 522, pp. 9-17, 2019.

[13] A. Candelieri, B. G. Galuzzi, I. Giordani, and F. Archetti, "Vulnerability of public transportation networks against directed attacks and cascading failures," Public Transport, vol. 11, no. 1, pp. 1-23, 2019.

[14] H. Liu, L. Zhao, R. Yin, X. Hao, Y. Li, and B. Liu, "A metric of topology fault-tolerance based on cascading failures for wireless sensor networks," Journal of Information and Computational Science, vol. 8, no. 14, pp. 3227-3237, 2011.

[15] R. Yin, B. Liu, and Y. Li, "The critical load of scale-free faulttolerant topology in wireless sensor networks for cascading 
failures," Physica A Statistical Mechanics and Its Applications, vol. 409, no. 3, pp. 8-16, 2014.

[16] Y. Li, R. Yin, and B. Liu, "Cascading failure research on scalefree fault tolerant topology in wireless sensor networks," Journal of Beijing University of Posts and Telecommunications, vol. 37, no. 2, pp. 74-78, 2014.

[17] Z. Ye, T. Wen, Z. Liu, X. Song, and C. Fu, "Fault-Tolerant scheme for cascading failure of scale-free wireless sensor networks," in Proceedings of the 2016 IEEE International Conference on Information and Automation, IEEE ICIA 2016, pp. 2006-2011, China, August 2016.

[18] X. Hu, W. Li, and X. Fu, "Analysis of cascading failure based on wireless sensor networks," in Proceedings of the IEEE International Conference on Systems, Man, and Cybernetics, SMC 2015, pp. 1279-1284, Hong Kong, October 2015.

[19] X. Fu, Y. Yang, and O. Postolache, "Invulnerability of clustering wireless sensor networks against cascading failures," IEEE Systems Journal, no. 99, pp. 1-12, 2018.

[20] X. Fu, H. Yao, and Y. Yang, "Modeling and analyzing cascading dynamics of the clustered wireless sensor network," Reliability Engineering and System Safety, vol. 186, pp. 1-10, 2019.

[21] X. Fu, H. Yao, and Y. Yang, "Modeling and analyzing the cascading invulnerability of wireless sensor networks," IEEE Sensors Journal, vol. 19, no. 11, pp. 4349-4358, 2019.

[22] C. Qiu, H. Shen, and K. Chen, "An energy-efficient and distributed cooperation mechanism for k-coverage hole detection and healing in WSNs," IEEE Transactions on Mobile Computing, vol. 17, no. 6, pp. 1247-1259, 2018.

[23] S. Jannu and P. K. Jana, "A grid based clustering and routing algorithm for solving hot spot problem in wireless sensor networks," Wireless Networks, vol. 22, no. 6, pp. 1901-1916, 2016.

[24] X. Deng, Z. Tang, L. T. Yang, M. Lin, and B. Wang, "Confident information coverage hole healing in hybrid industrial wireless sensor networks," IEEE Transactions on Industrial Informatics, vol. 14, no. 5, pp. 2220-2229, 2018.

[25] J. Ren, Y. Zhang, K. Zhang, A. Liu, J. Chen, and X. S. Shen, "Lifetime and energy hole evolution analysis in data-gathering wireless sensor networks," IEEE Transactions on Industrial Informatics, vol. 12, no. 2, pp. 788-800, 2016.

[26] H. Huang, H. Yin, G. Min, X. Zhang, W. Zhu, and Y. Wu, "Coordinate-assisted routing approach to bypass routing holes in wireless sensor networks," IEEE Communications Magazine, vol. 55, no. 7, pp. 180-185, 2017. 


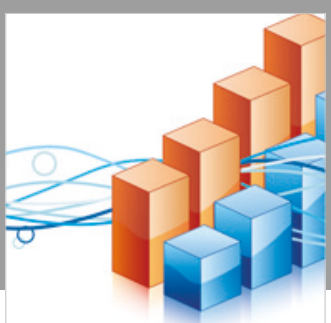

Advances in

Operations Research

\section{-n-m}
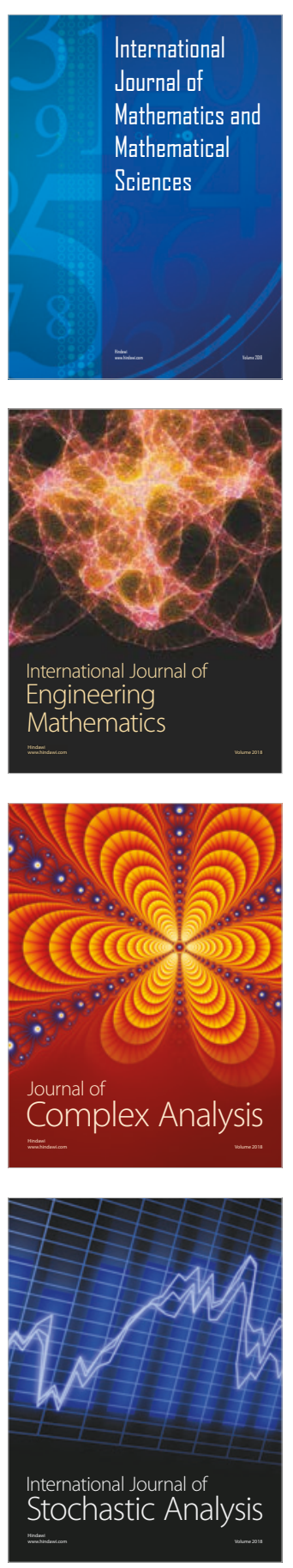
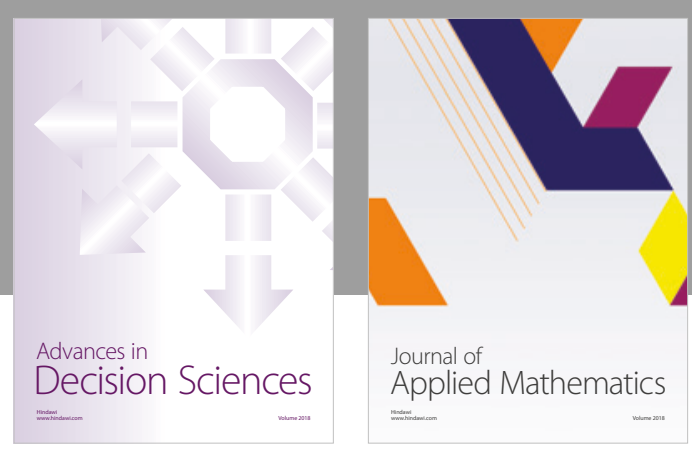

Journal of

Applied Mathematics
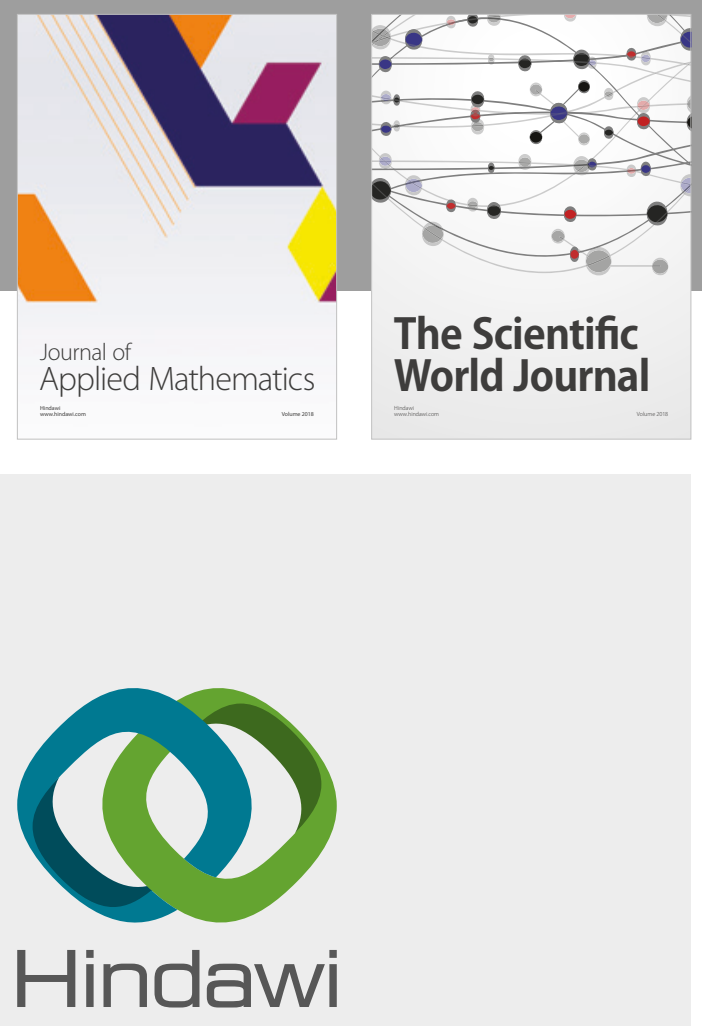

Submit your manuscripts at

www.hindawi.com

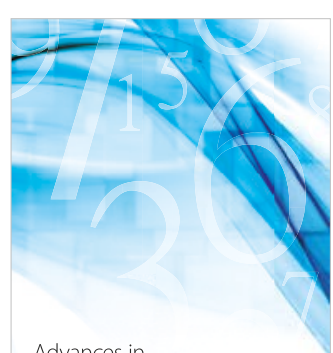

Advances in
Numerical Analysis
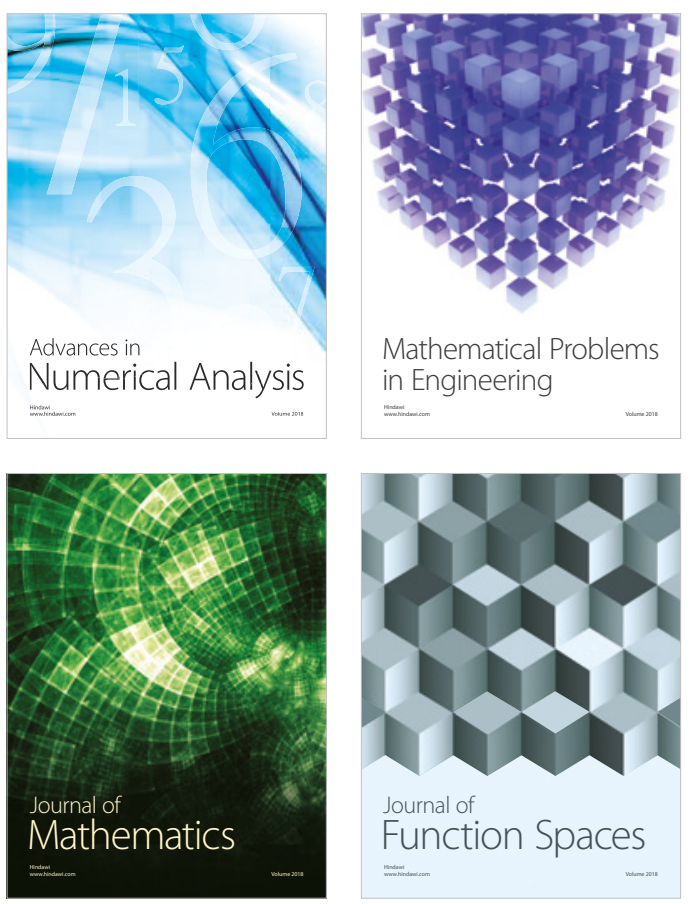

Mathematical Problems in Engineering

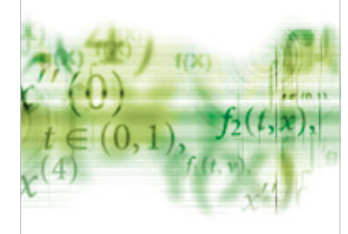

International Journal of

Differential Equations

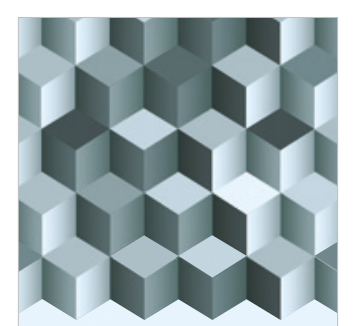

Journal of

Function Spaces
The Scientific

World Journal

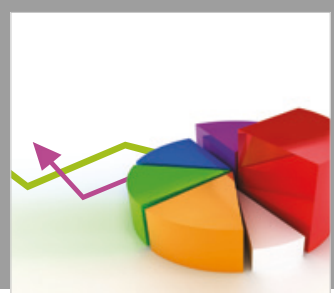

Journal of

Probability and Statistics
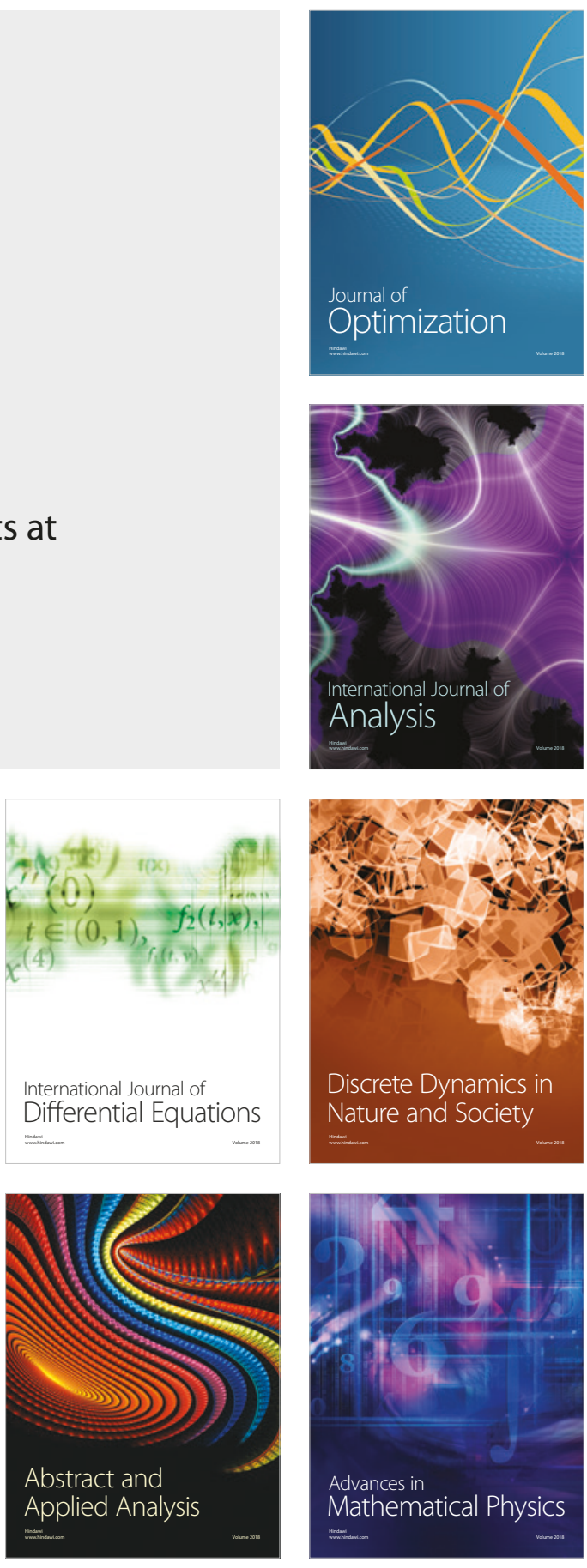